\title{
On Solution of Wave Propagation Control Problems using Conjugate Gradient Method of Higher - Order
}

\author{
${ }^{1}$ R.A. Raji and ${ }^{2}$ M.O. Oke \\ ${ }^{1}$ Department of Mathematics and Statistics, Osun State Polytechnic, P. M. B. 301, Iree, Osun State, Nigeria. \\ ${ }^{2}$ Department of Mathematical Sciences, Ekiti State University, P. M. B. 5363, Ado - Ekiti, Ekiti State, Nigeria.
}

\begin{abstract}
The conjugate gradient method is an algorithm for the numerical solution of systems of linear equations whose matrices are positive-definite and symmetric. It is an iterative method that can even be applied to solve a sparse system of equations. In this paper, we applied the conjugate gradient method of higher - order to wave propagation control problems. The algorithm of this method was implemented using MATLAB 7.10.0 codes to get numerical results.
\end{abstract}

Keywords: Wave Propagation, Optimal Control, Positive-definite matrix, Symmetric Matrix, Conjugate Gradient Method

\section{Introduction}

Optimal control deals with the problem of finding a control law for a given system such that a certain optimality criterion is achieved. The control problem includes a cost functional that is a function of state and control variables. An optimal control problem is a set of differential equations describing the paths of the control variables that minimize the cost functional. It can be derived using pontrygin's maximum principle or by solving Hamilton-Jacobi-Bellman equation. Wave equation is the simplest example of the hyperbolic equation of second order. If $x \in R^{n}$ represents the space variable and t the time variable, Zakharenko (2012) and Brillovin (1960) showed that the hyperbolic equation of second order can be used to model waves in pipes or vibrating strings when $n=1$; waves on the surface of water when $n=2$ and waves in optices or a coustics when $n=3$.

In this paper, we applied the conjugate gradient method of higher - order to the wave propagation control problems. Wave propagation problems are of great concern to scientists and engineers because the principle involved are common to many physical problems such as water waves in an ocean and acoustic or electromagnetic waves in a solid body, Zakharenko (2011) and Brillovin (1960).

A lot of research works have been carried out on solution of wave propagation equations. Banimok and David (2006) considered an iterative method for solving wave equations while Ogunmadewa (2009) worked on some numerical methods of solving wave equations. We also have some iterative methods for large linear systems of ocean and electromagnetic waves in Kincard and Haycs (1990). None of these researchers used the conjugate gradient method of higher - order in solving the wave propagation problems. Therefore, in this research work, we considered solution of wave propagation control problems using the algorithm of conjugate gradient method of higher - order. The algorithm was implemented using MATLAB 7.10.0 codes to get some numerical results.

\section{Materials and Methods}

In this paper we considered, in the first example, an optimal control problem of the form

$\operatorname{Min} J(z, u)=\int_{0}^{T} \int_{0}^{\sigma}\left[z^{2}(x, t)+u^{2}(x, t)\right] d x d t$

Subject to the I-D wave propagation equations

$$
\begin{aligned}
& \frac{\partial^{2} z}{\partial t^{2}}+a^{2} \frac{\partial^{2} z}{\partial \lambda^{2}}+u(x, t)=0 \\
& z(0, t)=z(\lambda, t)=0
\end{aligned}
$$


$z(x, 0)=z(\lambda, t)=0$

$z(x, 0)=z(x)$

where the control function $\mathrm{u}(\mathrm{x}, \mathrm{t})$ is being introduced in a similar fashion to the spring force in the generalized Lienard equation or the damping force associated with mechanical vibrations in resisting media.

In the second example, we looked at a problem of functional minimization on $R^{n}$ where $\mathrm{n}$ is very large and the matrix operator A is symmetric and positive definite. Since A is very large in size, we partitioned it for our convenience and then applied the algorithm of conjugate gradient method of higher - order to solve the problem. In general the minimization problem may not be in vector matrix notation form, we therefore present the third example in a simplified summation form and applied the algorithm of conjugate gradient method of higher order to solve it.

Below are some theorems which we are going to apply in solving some of the problems in this paper.

\section{Theorem 1}

Suppose $\mathrm{H}$ is an Hilbert space and $\mathrm{T}$ is a linear continuous operator defined on $\mathrm{H}$ with the map $T: H \rightarrow H$. If $x \in H$ and for fixed $y \in H$, we define $f_{y}(x)=\langle T x, y\rangle_{H}$, then $f_{y}$ is a continuous linear functional defined on $\mathrm{H}$.

Proof: Kesavan (1989) and Liusternik and Sobolev (1961) showed that since $f_{y}$ is a continuous linear functional on the Hilbert space $\mathrm{H}$, there is an element $y^{A}$ such that $f_{y}(x)=\left\langle x, y^{*}\right\rangle_{H}$. Now let $\langle T x, y\rangle_{H}$ with $\mathrm{T}$ self adjoint be interpreted as a functional of $\mathrm{x}$ and $\mathrm{y}$ variables and let us write $\langle T x, y\rangle_{H}=T(x, y)$, then

$T\left(\lambda x_{1}+\mu x_{2}, y\right)=\lambda T\left(x_{1}, y\right)+\mu T\left(x_{2}, y\right)$

It can easily be seen that

$T(x, y)=T(y, x)$.

A functional that satisfies the conditions in (6) and (7) above for any values of $\lambda$ and $\mu$ is called a bilinear

Hermitian form. This form is bounded provided $\mathrm{T}$ is bounded. The boundedness of $T(x, y)$ means

$$
\mid T(x, y) C_{T}\|\| x\left\|_{H}\right\| y \|_{H}
$$

where $C_{T}=\|T\|_{H}$ is a constant.

From the result due to Liusternik and Sobolev (1961), it follows that every self adjoint bounded operator T generates a bounded bilinear Hermitian form:

$T(x, y)=\|T x, y\|_{H}=\|x, T y\|_{H}$

and conversely. If a bounded bilinear Hermitian form $T(x, y)$ is given, then it defines a bounded self adjoint $\mathrm{T}$ satisfying:

$$
\|T x, y\|_{H}=T(x, y)=T(y, x)
$$

This implies that, if we fix y in $T(x, y)$, then by virtue of the fact that

$$
f_{y}(x)=\left\langle x, y^{*}\right\rangle_{H}
$$

we get

$T(x, y)=\left\langle x, y^{*}\right\rangle_{H}$

where y is uniquely determined.

\section{Theorem 2}

The functional $P\left(Z_{1}, Z_{2}\right)$ is a bounded, bilinear and self adjoint form. 
Proof: There exist $r>0$ which satisfies

$\langle Z, A Z\rangle \leq r\|Z\|^{2}$

where

$r=\max \left\{r_{1}>r_{2}, r_{3}\right\}$,

$r_{1}=a+\mu c^{2}+\mu|c|(1+|d|)$;

$r_{2}=b+\mu d^{2}+\mu|d|(1+|c|) ;$

and

$r_{3}=\mu(1+|c|+|d|)$;

which verifies the boundedness of $(Z, A Z)$.

The self adjointness of $P\left(Z_{1}, Z_{2}\right)$ follows directly from the fact that

$P\left(Z_{1}, Z_{2}\right)=P\left(Z_{2}, Z_{1}\right)$

and the bilinearity is as a consequence of the fact that the relation in (19) below holds:

$P\left(\lambda Z_{1}+\mu Z_{2}, u\right)=\lambda P\left(Z_{1}, u\right)+\mu P\left(Z_{2}, u\right)$

for any $\lambda \neq 0$ and $\mu \neq 0$.

\section{Example 1}

\section{Computational Examples and Results}

Let us consider the control problem

$\operatorname{Min} \int_{0}^{T} a x^{2}(t)+b u^{2}(t) d t$

where $\mathrm{a}$ and $\mathrm{b}$ are constants and $a>0, b>0$.

subject to:

$x^{\prime}(t)=c x(t)+d u(t)$

$0 \leq t \leq T$

We now associate with the unconstrained equivalence of this problem the control operator $\mathrm{A}$ in the following manner

$\operatorname{Min}\langle Z, A Z\rangle_{k} \equiv \int_{0}^{T} a x^{2}(t)+b u^{2}(t) d t+\mu\left\|x^{\prime}(t)-c x(t)-d u(t)\right\| d t$

$\equiv \int_{0}^{T}\left[a x^{2}(t)+b u^{2}(t) d t+\mu\left(x^{\prime}(t)-c x(t)-d u(t)\right)^{2}\right] d t$

Since $x \in R e^{\prime}{ }_{,} K=\left[H_{1}(0, T) X L_{2}(0, T)\right]$ and $\mathrm{Z}$ is the ordered pair $(\lambda, \mu), \mu>0$ is the penalty constant.

For our subsequent development, we set:

$Z_{1}=\left(x_{1}, u_{1}\right)$ and $Z_{2}=\left(x_{2}, u_{2}\right)$.

Thus, for convergence of the sequel, we associate with the problem, a functional $\mathrm{P}$ and a control operator $\mathrm{A}$ as follows

$P(x, u, \mu) \equiv \int_{0}^{T}\left[a x^{2}(t)+b u^{2}(t)+\mu\left(x^{\prime}(t)-c x(t)-d u(t)\right)^{2}\right] d t$

We can easily see that

$$
\begin{aligned}
& P(x, u, \mu) \equiv P\left(Z_{1}, Z_{2}\right) \equiv \\
& \int_{0}^{T}\left[a x_{1}(t) x_{2}(t)+b u_{1}(t) u_{2}(t)+\mu\left(x_{1}{ }^{\prime}(t) x_{2}{ }^{\prime}(t)+\mu c^{2} x_{1}(t) x_{2}(t)\right] d t\right. \\
& +\mu \int_{0}^{T}\left[d^{2} u_{1}(t) u_{2}(t)-c x_{1}{ }^{\prime}(t) x_{2}(t)-c x_{2}{ }^{\prime}(t) x_{1}(t)+c d x_{1}(t) u_{2}(t)\right] d t \\
& +\mu \int_{0}^{T}\left[c d x_{2}(t) u_{1}(t)-d x_{1}{ }^{\prime}(t) u_{2}(t)-d x_{2}{ }^{\prime}(t) u_{1}(t)\right] d t
\end{aligned}
$$


where the equivalence is preserved under the following presumed equivalence laws

$x_{1}(t) \equiv x_{2}(t) \equiv x(t), \quad 0 \leq t \leq T$

$x_{1}{ }^{\prime}(t) \equiv x_{2}{ }^{\prime}(t) \equiv x^{\prime}(t), 0 \leq t \leq T$

$u_{1}(t) \equiv u_{2}(t) \equiv u(t), \quad 0 \leq t \leq T$

The boundedness of $\left\lfloor P\left(Z_{1}, Z_{2}\right)\right\rfloor$, the bilinearity of $P\left(Z_{1}, Z_{2}\right)$ and it's self adjointness permits us to construct a unique control operator $\mathrm{A}$ that satisfies

$\langle Z, A Z\rangle \equiv \int_{0}^{T}\left[a x^{2}(t)+b u^{2}(t)+\mu\left(x^{\prime}(t)-c x(t)-d u(t)\right)^{2}\right] d t$, for $\mu>0$.

Example 2

Let us consider the case when $\mathrm{n}=1$

$F(x)=F_{0}+\langle a, x\rangle R^{n}+\frac{1}{2}\langle x, A x\rangle R^{n}$

with the following data:

$A=\left(\begin{array}{ll}P & Q \\ R & S\end{array}\right)$

where

$P=\left(\begin{array}{ccccccccccc}180 & -12 & 0 & 20 & 0 & 0 & 0 & -20 & 20 & 0 & 20 \\ 20 & 180 & -20 & -20 & 0 & 0 & 0 & 0 & 0 & 0 & 0 \\ 0 & -20 & 120 & 0 & -20 & 40 & 0 & 0 & 0 & 0 & 0 \\ 20 & -20 & 0 & 180 & 0 & -20 & 0 & 0 & 0 & 0 & -20 \\ 0 & 0 & -20 & 0 & 120 & 0 & 40 & 0 & 0 & 0 & -20 \\ 0 & 0 & 40 & -20 & 0 & 240 & 0 & 60 & 0 & 100 & 0 \\ 0 & 0 & 0 & 0 & 40 & 0 & 300 & 0 & 0 & 0 & 20 \\ -20 & 0 & 0 & 0 & 0 & 60 & 0 & 420 & 20 & 0 & 20 \\ 20 & 0 & 0 & 0 & 0 & 0 & 0 & -20 & 480 & -40 & 0 \\ 0 & 0 & 0 & 0 & 0 & 100 & 0 & 0 & -40 & 540 & 0 \\ 20 & 0 & 0 & -20 & -20 & 0 & 20 & 20 & 0 & 0 & 600\end{array}\right)$

$Q=\left(\begin{array}{ccccccccccc}-20 & 0 & -20 & 20 & 0 & 0 & -20 & -20 & 0 & 0 & 0 \\ 20 & 0 & 0 & -40 & 0 & -20 & 0 & -20 & 40 & 0 & 20 \\ 0 & 20 & 0 & 0 & -20 & 0 & 0 & 0 & 0 & 40 & 0 \\ 0 & -20 & 0 & 0 & 0 & -40 & 20 & 0 & 20 & 0 & 40 \\ 20 & 0 & -20 & 20 & 20 & 0 & 0 & 40 & 0 & 0 & 0 \\ 20 & 0 & 40 & 0 & 0 & 0 & 20 & 0 & 0 & -20 & 0 \\ 0 & 20 & 0 & 0 & -40 & 0 & 0 & 0 & -20 & 0 & -60 \\ 0 & 0 & 0 & -20 & 0 & -20 & 0 & -20 & 40 & 0 & 0 \\ -20 & 0 & 0 & 0 & 0 & 0 & -20 & 0 & 0 & 20 & -20 \\ 0 & 0 & 20 & 0 & 20 & 0 & 0 & 0 & 20 & 0 & 0 \\ 0 & 20 & 0 & 40 & 0 & 0 & 0 & 20 & 0 & 0 & -20\end{array}\right)$

$R=\left(\begin{array}{ccccccccccc}-20 & 20 & 0 & 0 & 20 & 20 & 0 & 0 & -20 & 0 & 0 \\ 0 & 0 & 20 & -20 & 0 & 0 & 20 & 0 & 0 & 0 & 20 \\ -20 & 0 & 0 & -20 & 40 & 0 & 0 & 0 & 0 & -20 & 0 \\ 20 & 40 & 0 & 0 & 20 & 0 & 0 & 20 & 0 & 0 & 40 \\ 0 & 0 & -20 & 0 & 20 & 0 & -40 & 0 & 0 & 20 & 0 \\ 0 & -20 & 0 & -40 & 0 & 0 & 0 & -20 & 0 & 0 & 0 \\ -20 & 0 & 0 & 20 & 0 & 20 & 0 & 0 & -20 & 0 & 0 \\ -20 & 20 & 0 & 0 & 40 & 0 & 0 & 20 & 0 & 0 & 20 \\ 0 & 40 & 0 & 20 & 0 & 0 & -20 & 40 & 0 & 20 & 0 \\ 0 & 0 & 40 & 0 & 0 & -20 & 0 & 0 & 20 & 0 & 0 \\ 0 & 20 & 0 & 40 & 0 & 0 & -60 & 0 & -20 & 0 & -20\end{array}\right)$ 
On Solution of Wave Propagation Control Problems using Conjugate Gradient Method of Higher.....

$S=\left(\begin{array}{ccccccccccc}720 & 0 & 40 & 0 & 0 & 20 & -20 & 0 & 0 & -40 & 0 \\ 0 & 780 & 40 & 0 & 0 & 20 & -20 & 0 & -20 & 0 & 0 \\ 40 & 0 & 840 & 0 & 0 & -20 & -20 & 20 & 0 & 0 & 40 \\ 0 & 0 & 0 & 900 & 0 & 0 & 0 & 20 & 0 & 20 & 0 \\ 0 & -40 & 0 & 0 & 960 & 40 & 20 & 0 & 20 & 0 & 0 \\ 20 & 0 & -20 & 0 & 40 & 1020 & 0 & 0 & 0 & 40 & 0 \\ -20 & 0 & -20 & 0 & 20 & 0 & 1080 & -20 & 0 & 0 & 20 \\ 0 & 0 & 20 & 20 & 0 & 0 & -20 & 1140 & 0 & 20 & 0 \\ 0 & -20 & 0 & 0 & 20 & 0 & 0 & 0 & 1200 & 0 & 40 \\ -40 & 0 & 0 & 20 & 0 & 40 & 0 & 20 & 0 & 1260 & 0 \\ 0 & 0 & 40 & 0 & 0 & 0 & 20 & 0 & 40 & 0 & 1320\end{array}\right)$

In this example

$\vec{a}=(2.00,2.50,3.50,-2.00,3.00,4.50,2.50,3.50,2.50,1.00,0.00$,

$2.00,3.00,2.50,2.00,4.00,3.50,2.00,1.00,-2.50,3.50,5.00$ )

The solution vector $\vec{x}$ was computed via the conjugate gradient method and we obtain the following results

$\vec{x}=(-0.053,-0.0192,-0.329,0.093,-0.0274,-0.0112,-0.0058,-0.0083$,

$-0.0049,-0.0035,-0.0005,-0.0016,-0.0028,-0.0032,-0.0023,-0.0046$,

$-0.0034,0.0021,-0.006,0.0029,-0.0017,-0.0041$ ).

Example 3

Minimize $Q+\langle a, x\rangle$

where $Q=\sum_{i=1}^{35} R_{i}$

and

$R_{1}=132 x_{1}^{2}-11 x_{1} x_{2}+11 x_{1} x_{4}+11 x_{1} x_{8}+11 x_{1} x_{9}+11 x_{1} x_{10}+11 x_{1} x_{12}$

$R_{2}=-11 x_{1} x_{14}+11 x_{1} x_{15}+11 x_{1} x_{18}+11 x_{1} x_{19}+11 x_{1} x_{23}+11 x_{2} x_{1}+132 x_{2}{ }^{2}$

$R_{3}=-11 x_{2} x_{3}+11 x_{2} x_{4}+11 x_{2} x_{12}+22 x_{2} x_{15}+11 x_{2} x_{19}+22 x_{2} x_{20}$

$R_{4}=-11 x_{2} x_{22}+11 x_{2} x_{24}+11 x_{2} x_{25}+11 x_{3} x_{2}+88 x_{3}{ }^{2}-11 x_{3} x_{5}+22 x_{3} x_{6}$

$R_{5}=-11 x_{3} x_{13}-11 x_{3} x_{16}+22 x_{3} x_{21}+11 x_{3} x_{23}+11 x_{4} x_{1}+11 x_{4} x_{2}+132 x_{4}{ }^{2}$

$R_{6}=-11 x_{4} x_{6}-11 x_{4} x_{11}-11 x_{4} x_{13}-22 x_{4} x_{17}+11 x_{4} x_{18}+11 x_{4} x_{20}+22 x_{4} x_{22}$

$R_{7}=11 x_{4} x_{24}-11 x_{5} x_{3}+88 x_{5}^{2}+22 x_{5} x_{7}-11 x_{5} x_{11}+11 x_{5} x_{12}+11 x_{5} x_{14}$

$R_{8}=11 x_{5} x_{15}+11 x_{5} x_{16}+22 x_{5} x_{19}+22 x_{5} x_{23}+33 x_{5} x_{25}+22 x_{6} x_{3}-11 x_{6} x_{4}$

$R_{9}=176 x_{6}{ }^{2}+33 x_{6} x_{8}+55 x_{6} x_{10}+11 x_{6} x_{12}+22 x_{6} x_{14}+11 x_{6} x_{18}-11 x_{6} x_{21}$

$R_{10}=22 x_{7} x_{5}+220 x_{7}^{2}+11 x_{7} x_{11}+11 x_{7} x_{13}-22 x_{7} x_{16}-11 x_{7} x_{20}-33 x_{7} x_{22}$

$R_{11}=-11 x_{8} x_{1}+33 x_{8} x_{6}+308 x_{8}{ }^{2}-11 x_{8} x_{9}+11 x_{8} x_{11}-11 x_{8} x_{15}-11 x_{8} x_{17}$

$R_{12}=-11 x_{8} x_{19}+22 x_{8} x_{20}+22 x_{8} x_{24}+11 x_{9} x_{1}-11 x_{9} x_{8}+352 x_{9}{ }^{2}-22 x_{9} x_{10}$

$R_{13}=-11 x_{9} x_{12}-11 x_{9} x_{18}+11 x_{9} x_{21}-11 x_{9} x_{22}+11 x_{9} x_{25}+55 x_{10} x_{6}-22 x_{10} x_{9}$

$R_{14}=39 x_{10}{ }^{2}-11 x_{10} x_{14}+11 x_{10} x_{16}+11 x_{10} x_{20}-33 x_{10} x_{23}+11 x_{11} x_{1}-11 x_{11} x_{4}$

$R_{15}=-11 x_{11} x_{5}+11 x_{11} x_{7}+11 x_{11} x_{8}+440 x_{11}^{2}+11 x_{11} x_{13}+22 x_{11} x_{15}-11 x_{11} x_{18}$

$R_{16}=-11 x_{11} x_{22}+11 x_{11} x_{24}-11 x_{12} x_{1}+11 x_{12} x_{2}+11 x_{12} x_{5}+11 x_{12} x_{6}-11 x_{12} x_{9}$

$R_{17}=528 x_{12}^{2}+22 x_{12} x_{14}+11 x_{12} x_{17}-11 x_{12} x_{18}-22 x_{12} x_{21}-22 x_{12} x_{25}+11 x_{13} x_{3}$

$R_{18}=-11 x_{13} x_{4}+11 x_{13} x_{7}+11 x_{13} x_{11}+572 x_{13}{ }^{2}-22 x_{13} x_{16}-11 x_{13} x_{20}+11 x_{13} x_{23}$

$R_{19}=-11 x_{14} x_{1}-11 x_{14} x_{5}+22 x_{14} x_{6}-11 x_{14} x_{10}+22 x_{14} x_{12}+616 x_{14}{ }^{2}-11 x_{14} x_{17}$

$R_{20}=-11 x_{14} x_{18}+11 x_{14} x_{19}+22 x_{14} x_{22}+11 x_{15} x_{1}-22 x_{15} x_{2}+11 x_{15} x_{5}-11 x_{15} x_{8}$

$R_{21}=22 x_{15} x_{11}+660 x_{15}{ }^{2}+11 x_{15} x_{19}+11 x_{15} x_{21}-33 x_{15} x_{24}-11 x_{16} x_{3}+11 x_{16} x_{5}$ 


$$
\begin{aligned}
& R_{22}=-22 x_{16} x_{7}+11 x_{16} x_{10}-22 x_{16} x_{13}+704 x_{16}{ }^{2}+22 x_{16} x_{17}+11 x_{16} x_{18}+11 x_{16} x_{20} \\
& R_{23}=-22 x_{16} x_{23}-11 x_{16} x_{25}-11 x_{17} x_{2}-22 x_{17} x_{4}-11 x_{17} x_{8}+11 x_{17} x_{12}-11 x_{17} x_{14} \\
& R_{24}=22 x_{17} x_{16}+748 x_{17}{ }^{2}+22 x_{17} x_{21}-11 x_{17} x_{23}-11 x_{18} x_{1}+11 x_{18} x_{4}+11 x_{18} x_{6} \\
& R_{25}=-11 x_{18} x_{19}-11 x_{18} x_{12}-11 x_{18} x_{14}+11 x_{18} x_{16}+792 x_{18}{ }^{2}-11 x_{18} x_{19}+11 x_{18} x_{22} \\
& R_{26}=-11 x_{19} x_{1}-11 x_{19} x_{2}+22 x_{19} x_{5}-11 x_{19} x_{8}+11 x_{19} x_{11}+11 x_{19} x_{14}-11 x_{19} x_{15} \\
& R_{27}=-11 x_{19} x_{18}+836 x_{19}{ }^{2}+11 x_{19} x_{21}-11 x_{19} x_{24}+22 x_{20} x_{2}+11 x_{20} x_{4}-11 x_{20} x_{7} \\
& R_{28}=22 x_{20} x_{8}+11 x_{20} x_{10}-11 x_{20} x_{13}+880 x_{20}{ }^{2}+22 x_{20} x_{22}+11 x_{20} x_{23} \\
& R_{29}=-22 x_{20} x_{25}+22 x_{21} x_{3}-11 x_{21} x_{6}+11 x_{21} x_{9}-22 x_{21} x_{12}+11 x_{21} x_{15}+22 x_{21} x_{17} \\
& R_{30}=11 x_{21} x_{9}+924 x_{21}{ }^{2}-22 x_{21} x_{24}+11 x_{22} x_{2}+22 x_{22} x_{4}-33 x_{22} x_{25}+11 x_{23} x_{1} \\
& R_{31}=-11 x_{22} x_{11}+22 x_{22} x_{14}+11 x_{22} x_{18}+22 x_{22} x_{20}+924 x_{22}{ }^{2}+11 x_{22} x_{25}+11 x_{23} x_{1} \\
& R_{32}=11 x_{23} x_{3}+22 x_{23} x_{5}-33 x_{23} x_{10}+11 x_{23} x_{13}-22 x_{23} x_{16}-11 x_{23} x_{17}+11 x_{23} x_{20} \\
& R_{33}=101 x_{23}{ }^{2}+11 x_{24} x_{2}+11 x_{24} x_{4}+22 x_{24} x_{8}+11 x_{24} x_{11}-33 x_{24} x_{15}-11 x_{24} x_{19} \\
& R_{34}=-22 x_{24} x_{21}+1056 x_{24}{ }^{2}-11 x_{25} x_{2}+33 x_{25} x_{5}+11 x_{25} x_{9}-22 x_{25} x_{12}-11 x_{25} x_{16} \\
& R_{35}=-22 x_{25} x_{20}+11 x_{25} x_{22}+1100 x_{25}{ }^{2} \\
&
\end{aligned}
$$

In this example

$$
\vec{a}=(4.00,5.00,9.00,-4.00,8.00,5.00,3.00,4.50,8.00,-5.00,2.00,7.00 \text {, }
$$$$
14.00,3.00,7.50,5.00,4.50,14.00,6.50 \text {, }-2.00,9.00,7.00,11.00,16.50,10.00 \text { ) }
$$

On applying the conjugate gradient method algorithm in solving this problem, we obtained convergence to the optimal solution $\vec{x} \in R^{25}$ after 24 iterations. This is an iteration less than the dimensionality of the space on which the problem was defined. The optimal solution is therefore given by $\vec{x}=(-0.0381,-0.0492,-0.1132,0.0293,-0.0953,-0.0111,-0.0046,-0.0154$, $-0.0220,0.0124,-0.0033,-0.0119,-0.0217,-0.0063,-0.0115,-0.0083,-0.0057,-0.0189$, $-0.0067,0.0032,-0.0072,-0.0074,-0.0068,-0.0156,-0.0067)$.

\section{Conclusion}

In this paper, we used theorems 1 and 2 to show the existence of solution for the wave propagation control problem in the first example. We now applied the conjugate gradient method algorithm to solve largescale problems involving wave propagation control in the second and third example. The algorithm was implemented using MATLAB 7.10.0 codes and the numerical results obtained are given in the examples. In the second example, we obtained convergence to the optimal solution $\vec{x} \in R^{22}$ after 21 iterations and in the third example; we obtained convergence to the optimal solution $\vec{x} \in R^{25}$ after 24 iterations. These iterations are less than the dimensionality of the space on which the problems were defined. This is one of the remarkable properties of the conjugate gradient method algorithm that we applied.

\section{References}

[1]. Zakharenko, A.A. Thirty Two New SH-Waves Propagating in PEM Plates of Class 6 mm, Saarbruecken - Krasnoyarsk, LAP LAMBERT Academic Publishing GmbH \& Co., 2012.

[2]. Banimok, J.A. and David K.R. An Iterative Method for Solving Wave Equations, PENTUM Journal of Mathematics and Computer Science, Vol. 5, 2006, 38-52.

[3]. Brillovin, L. Wave Propagation and Group Velocity, Academic press, New York, 1960.

[4]. Zakharenko, A.A. Seven New SH-Saws in Cubic Piezoelectromagnetics, Saarbruecken - Krasnoyarsk, LAP LAMBERT Academic Publishing GmbH \& Co., 2011.

[5]. Kesavan, S. Topics in Functional Analysis and Applications, John willey, New York, 1989.

[6]. Liusternik, L.A. and Sobolev, V.I. Elements of Functional Analysis, Frederick Ungar, New York, 1961.

[7]. Kincard, D.R. and Haycs, L.J. Iterative Methods for Large Linear Systems, Academic press, Inc., New York, 1990.

[8]. Ogunmadewa J.O. Some Numerical Methods of Solving Wave Equations, MAVIC Journal of Sciences, Vol. 3, 2009, 106-119. 